\title{
Aspectos psicológicos e psicossociais em indivíduos vivendo com HIV/AIDS
}

\author{
Psychological and psychosocial aspects in people living with HIVIAIDS
}

\author{
Marcelo Comerlato Scotta $a^{1} \bowtie$ \\ ${ }^{1}$ Faculdade de Medicina da Pontifícia Universidade Católica do Rio Grande do Sul
}

DESCRITORES: soropositividade para HIV; infecções por HIV; antirretrovirais; fenômenos e processos psicológicos; depressão; apoio social.

KEY WORDS: HIV seropositivity; HIV infections; anti-retroviral agents; psychological phenomena and processes; depression; social support.

A infecção pelo HIV afeta cerca de 30 milhões de indivíduos no mundo, estando a maioria desses pacientes nos países em desenvolvimento. Com poucos paralelos na história da medicina, esta doença teve sua história natural radicalmente modificada pelo uso da terapia antirretroviral mais potente, com grande redução de morbimortalidade e aquisição de caráter crônico da infecção [1].

Neste número, a Scientia Medica apresenta dois estudos que abordam os aspectos psicossociais e as comorbidades psiquiátricas de indivíduos infectados pelo HIV. O estudo realizado por dos Santos et al. [2] no Hospital Universitário de Santa Maria, Rio Grande do Sul, identificou associação entre sentimento de autoeficácia e maior suporte social em pacientes cronicamente infectados, de cor negra e escolaridade inferior a sete anos. Evidências apontam para a importância de identificar o sentimento de autoeficácia em pacientes com HIV, visto que este e, consequentemente, a adesão ao tratamento, melhoram quando os profissionais de saúde estão engajados com esses aspectos [2,3].
Em relação aos transtornos depressivos, Freitas et al. [4] encontraram prevalência de $65,5 \%$ de sintomas característicos em pacientes em acompanhamento no Hospital do Porto, em Portugal, sendo $32,7 \%$ classificados como graves, em população com elevada prevalência de drogadição, que parece ser semelhante à reportada em serviços de saúde brasileiros [4-6].

A interação entre a infecção pelo HIV e comorbidades psiquiátricas é complexa e deletéria. Por um lado, o risco de aquisição e transmissão da infecção pelo HIV aumenta no paciente drogadito, com pouco suporte social e/ou comorbidades psiquiátricas associadas. Por outro, os fatores acima, se não diagnosticados e tratados adequadamente, podem interferir com a adesão à terapia antirretroviral e aumentar o risco de complicações e óbito secundários ao HIV. Devido a esse contexto, a adequada caracterização do impacto das comorbidades psiquiátricas na epidemia do HIV é de fundamental importância [7].

\section{REFERÊNCIAS}

1. Murray CJ, Ortblad KF, Guinovart C, Lim SS, Wolock TM, Roberts DA, et al. Global, regional, and national incidence and mortality for HIV, tuberculosis, and malaria during 1990-2013: a systematic analysis for the Global Burden of Disease Study 2013. Lancet. 2014;384(9947):1005-70. http://dx.doi.org/10.1016/S0140-6736(14)60844-8

2. dos Santos WM, Padoin SMM, Magnano TSBS, Dalmolin GL, Zuge SS. Fatores intervenientes entre suporte social e autoeficácia em pessoas infectadas pelo HIV. Sci Med. 2015;25(2):ID21105. http://dx.doi.org/10.15448/1980-6108.2015.2.21105

3. Chen WT, Wantland D, Reid P, Corless IB, Eller LS, lipinge S, et al. Engagement with health care providers affects self- efficacy, self-esteem, medication adherence and quality of life in people living with HIV. J AIDS Clin Res. 2013;4(11):256. http://dx.doi.org/10.4172/21556113.1000256 
4. Freitas P, Fernandes A, Morgado P. Depressão em pacientes HIV positivos: a realidade de um hospital português. Sci Med. 2015;25(2):ID20469. http://dx.doi.org/10.15448/1980-6108.2015.2.20469

5. Dal-Bo MJ, Manoel AL, Filho AO, Silva BQ, Cardoso YS, Cortez J, et al. Depressive symptoms and associated factors among people living with HIV/AIDS. J Int Ass Prov AIDS Care. 2015;14(2):136-40. http://dx.doi.org/10.1177/2325957413494829

6. Silveira MP, Guttier MC, Pinheiro CA, Pereira TV, Cruzeiro AL, Moreira LB. Depressive symptoms in HIV-infected patients treated with highly active antiretroviral therapy. Rev Bras Psiquiatr. 2012;34(2):162-7. http://dx.doi.org/10.1590/S1516-44462012000200008

7. Uthman OA, Magidson JF, Safren SA, Nachega JB. Depression and adherence to antiretroviral therapy in low-, middle- and high-income countries: a systematic review and meta-analysis. Current HIV/AIDS reports. 2014;11(3):291-307. http://dx.doi.org/10.1007/s11904-014$0220-1$ 